\title{
BLASCHKE QUOTIENTS AND NORMALITY
}

JOSEPH A. CIMA AND PETER COLWELL

Let $f$ be meromorphic in the unit disc $D$ and call $f$ normal if the family $\left\{f \circ S_{\alpha}\right\}$ is normal, where $\left\{S_{\alpha}\right\}$ represents the family of one to one conformal mappings of $D$ onto $D$. Further, if $\left\{z_{n}\right\}$ is a sequence in $D$ and $w=\left\{w_{1}, w_{2}, w_{3}, \cdots\right\}$ is a vector in $l^{\infty}$ we say that $\left\{z_{n}\right\}$ interpolates if there is a function in $H^{\infty}$ with $f\left(z_{n}\right)=w_{n}, n=1,2$, $3, \cdots$. A criterion that $\left\{z_{n}\right\}$ interpolate was given by L. Carleson $[2]$ as

$$
A(n)=\prod_{k=1 ; k \neq n}^{\infty}\left|\left(z_{n}-z_{k}\right) /\left(1-\bar{z}_{k} z_{n}\right)\right| \geqq \delta>0
$$

all $n=1,2,3, \cdots$. Our first result states that if $\left\{z_{n}\right\}$ interpolates then the Blaschke product for $\left\{z_{n}\right\}$, written as $B\left(z ; z_{n}\right)$, has its modulus greater than some positive number $\eta$ for points not close to $\left\{z_{n}\right\}$.

More precisely we let $\psi\left(z, z_{n}\right)=\left|\left(z-z_{n}\right) /\left(1-\bar{z}_{n} z\right)\right|$ be the pseudohyperbolic distance in the disc of $z$ to $z_{n}$. Then we have

Theorem 1. If $\left\{z_{n}\right\}$ interpolates and $B(z)=B\left(z ; z_{n}\right)$ is the Blaschke product for $\left\{z_{n}\right\}$ then for each $\epsilon>0$ there is an $\eta>0$ such that $|B(z)| \geqq \eta$ whenever $\psi\left(z, z_{n}\right) \geqq \epsilon, n=1,2,3, \cdots$.

Proof. Assume there is a sequence $\left\{t_{k}\right\}$ in $D$ and $\epsilon_{0}>0$ so that $\psi\left(t_{k}, z_{n}\right) \geqq \epsilon_{0}$ all $k$ and $n$ and that $B\left(t_{k}\right)$ tends to zero as $k$ tends to infinity. By a result of $A$. T. Cargo [1, p. 142] we may select a subsequence of $\left\{t_{k}\right\}$, which we again write as $\left\{t_{k}\right\}$, so that the Blaschke product $A(z)=A\left(z ; t_{k}\right)$ formed from the sequence $\left\{t_{k}\right\}$ has the property that

$$
\left|A\left(z_{n}\right)\right| \geqq a>0, \quad n=1,2,3, \cdots .
$$

We have then

$$
|A(z)|+|B(z)|>0, \quad z \in D .
$$

Then as in Theorem 1 of [3] we can find functions $g_{1}(z)$ and $g_{2}(z)$ holomorphic in $D$ and satisfying

$$
A(z) g_{1}(z)+B(z) g_{2}(z)=1, \quad z \in D .
$$

The sequence $\left\{\left(A\left(z_{n}\right)\right)^{-1}\right\}$ is in $l^{\infty}$ so there is an $f_{1}$ in $H^{\infty}$ satisfying $f_{1}\left(z_{n}\right)=\left(A\left(z_{n}\right)\right)^{-1}, n=1,2,3, \cdots$. Also we have that

Received by the editors March 27, 1967. 


$$
\left(f_{1}(z)-g_{1}(z)\right) \cdot(B(z))^{-1}=h(z)
$$

with $h$ holomorphic in $D$.

Define a holomorphic function $f_{2}$ on $D$ as follows:

Then

$$
f_{2}(z)=g_{2}(z)-h(z) A(z) .
$$

and

$$
f_{1}(z) A(z)+f_{2}(z) B(z)=1, \quad z \in D,
$$

$$
f_{2}(z)=\left(1-f_{1}(z) A(z)\right) \cdot(B(z))^{-1}
$$

is a bounded holomorphic function. Evaluating $f_{2}$ on the sequence $\left\{t_{n}\right\}$ shows it is unbounded, which is a contradiction, and so we have the result.

If we are given sequences of distinct points $A_{1}=\left\{\alpha_{n}\right\}$ and $A_{2}=\left\{\beta_{n}\right\}$ in $D$ with corresponding Blaschke products $B_{1}(z)=B\left(z ; \alpha_{n}\right)$ and $B_{2}(z)=B\left(z ; \beta_{n}\right)$, we have the following theorem concerning the normality of the quotient $B_{1}(z) \cdot\left(B_{2}(z)\right)^{-1}$.

THEOREM 2. If $A_{1}$ and $A_{2}$ are disjoint interpolating sequences in $D$, then the meromorphic function $f(z)=B_{1}(z) \cdot\left(B_{2}(z)\right)^{-1}$ is normal in $D$ if and only if $A_{1} \cup A_{2}$ interpolates.

Proof. Assume first $f(z)=B_{1}(z)\left(B_{2}(z)\right)^{-1}$ is normal in $D$. A criterion of O. Lehto and K. I. Virtanen [4, pp. 55-56] states that

$$
\rho(f) \leqq C|d z| /\left(1-|z|^{2}\right), \quad|z|<1,
$$

where $\rho$ is the spherical derivative of $f$ and $C$ is a positive constant. Evaluating this inequality on the points $\left\{\alpha_{j}\right\}$ of $A_{1}$ yields

$$
\left|B_{2}\left(\alpha_{i}\right)\right| \geqq(1 / C)\left|B_{1}^{\prime}\left(\alpha_{j}\right)\right|\left(1-\left|\alpha_{j}\right|^{2}\right) \text {. }
$$

We denote the partial product

$$
\frac{\left|\alpha_{j}\right|}{-\alpha_{j}}\left(\frac{1-\bar{\alpha}_{j} z}{z-\alpha_{j}}\right) B_{1}(z) \equiv \prod_{n=1 ; n \neq j}^{\infty} \frac{-\alpha_{n}}{\left|\alpha_{n}\right|}\left(\frac{z-\alpha_{n}}{1-\bar{\alpha}_{n} z}\right)
$$

as $\prod_{j}(z)$ and write $B_{1}(z)$ as

$$
B_{1}(z)=\frac{-\alpha_{j}}{\left|\alpha_{j}\right|}\left(\frac{z-\alpha_{j}}{1-\bar{\alpha}_{j} z}\right) \cdot \prod_{j}(z) .
$$

The condition of L. Carleson quoted previously shows that $\left|\prod_{j}\left(\alpha_{j}\right)\right|$ $\geqq \delta>0$. This shows that

$$
\lim \inf \left|B_{2}\left(\alpha_{j}\right)\right| \geqq(1 / C) \delta>0 .
$$

Thus $A_{1} \cup A_{2}$ in terpolates. 
Now assume $A_{1} \cup A_{2}$ interpolates. If the set of numbers

$$
\left|f^{\prime}(z)\right|\left(1-|z|^{2}\right) /\left(1+|f(z)|^{2}\right)
$$

were unbounded there must exist a sequence $\left\{z_{k}\right\}$ in $D$ such that

$$
\left|B_{1}\left(z_{k}\right)\right|^{2}+\left|B_{2}\left(z_{k}\right)\right|^{2} \rightarrow 0 .
$$

as $k$ tends to infinity. By Theorem 1 it must be the case that for some subsequence of $A_{1}$, say $\left\{\alpha_{k_{n}}\right\}$, we have $\psi\left(z_{k_{n}}, \alpha_{k_{n}}\right)$ tends to zero. Also there must be a subsequence $\left\{\beta_{j_{k_{n}}}\right\}$ with $\psi\left(z_{j_{k_{n}}}, \beta_{j_{k_{n}}}\right)$ tending to zero. This shows that $\psi\left(\alpha_{j_{k_{n}}}, \beta_{j_{k_{n}}}\right)$ tends to zero which is a contradiction to $A_{1} \cup A_{2}$ interpolating. The spherical derivative is bounded by a constant times $\left(1-|z|^{2}\right)^{-1}|d z|$ and so $f$ is normal.

We point out that $\rho(f)=\rho(1 / f)$ so that our Theorem 2 can be stated for $B_{2}(z) \cdot\left(B_{1}(z)\right)^{-1}$. The referee has pointed out the following equivalence.

TheOREM 3. If $A_{1}$ and $A_{2}$ are disjoint interpolating sequences in $D$, then $B_{1}(z) \cdot\left(B_{2}(z)\right)^{-1}$ is normal if and only if $B_{1}$ and $B_{2}$ are in no proper ideal of $H^{\infty}$.

Proof. If the ideal generated by $B_{1}$ and $B_{2}$ is not proper there are functions $f$ and $g$ in $H^{\infty}$ satisfying

$$
f(z) B_{1}(z)+g(z) B_{2}(z)=1, \quad z \in D .
$$

Setting $h_{1}(z)=f(z) B_{1}(z)$ and $h_{2}(z)=g(z) B_{2}(z)$ we observe that $h_{1}$ is zero on $A_{1}$ and one on $A_{2}$. Similarly $h_{2}$ is one on $A_{1}$ and zero on $A_{2}$. Using these functions and the hypothesis that $A_{1}$ and $A_{2}$ interpolate, we see that $A_{1} \cup A_{2}$ interpolates.

Conversely, if $A_{1} \cup A_{2}$ is interpolating then there is an $f \in H^{\infty}$ with $f B_{1}=1$ on $A_{2}$. Hence $1-f B_{1}$ is divisible by $B_{2}$ and thus one is in the ideal generated by $B_{1}$ and $B_{2}$.

\section{REFERENCES}

1. G. T. Cargo, Normal functions, the Montel property, and interpolation in $H^{\infty}$, Michigan Math. J. 10 (1963), 141-146.

2. L. Carleson, An interpolation problem for bounded analytic functions, Amer. J. Math. 80 (1958), 921-930.

3. J. A. Cima and G. D. Taylor, On the equation $f_{1} g_{1}+f_{2} g_{2}=1$ in $H^{\rho}$, Illinois J. Math. (to appear).

4. O. Lehto and K. I. Virtanen, Boundary behavior and normal meromorphic functions, Acta Math. 97(1957), 47-65.

University of North Carolina and Iowa State University 